\title{
The Effect of the Change of Solar-activity to Meteorological Phenomena
}

\author{
By K. Takahashi \\ Meteorological Research Institute, Tokyo \\ (Manuscript received 24 March, 1958)
}

\begin{abstract}
Tidal oscillations on the sun due to planets were calculated, and the following periods were obtained.

25 days, $1.475,2.375,2.890,3.893,6.552,9.592$, months. Such periodicities were detected in the change of solar activity as well as in some meteorological elements on the earth. It must be noticed, however, that the phase angles of the periodicities change regularly due to the 11 years cycle of the sunspot number.

Such periodic changes in the meteorological elements were correlated with those in the solar activity and the effects of the change of solar activity to meteorological phenomena were confirmed though the physical process of the effects was not yet clear. Of course, the amplitudes of such oscillations are very small, being estimated to be of the order of $10^{-4}$ times the absolute values. Therefore, they are insignificant in daily change of meteorological phenomena but can not be neglected for changes of long periods.

Such oscillation on the earth is modified by the general circulation of the atmosphere. The periodicities produced by the coupling between the solar effects and the annual change of the general circulation were calculated for a simple model. Some of the periodicities obtained by such calculations were detected in certain meteorological elements.
\end{abstract}

\section{Introduction}

The effect of the change of solar activity to meteorological phenomena is one of the long established research item and a lot of investigations have been made on it by many authors. Among them, the elaborate works by H. H. Clayton [1] are famous though his conclusion is not accepted fully. It seems to the present author, however, his anticipation is right as a whole though his analysis was criticized owing to the insufficiency in the test of significance.

In these few years, the present problem made new development with the progress of the researches on the physics of the upper atmosphere. R. A. Craig [3] and R. Shapiro [4] found the correlation between the meteorological elements and the change of the earth magnetism. A cross field semniar was held in 1955 at University of Cololado [5] with the participation of meteorologists and astronomers and there, it was concluded that corpuscular flow ejected from the sun seems to be important as the origin of the solar effect on the earth's atmosphere.

The present author has made also some investigations on the problem and has proposed a hypothesis [6] that the tidal oscillation on the sun caused by the planets will change the solar activity which will reflect on the meteorological phenomena of the earth. Of course, the effect of the change of the solar activity to the atmosphere must be very small. According to the present author's estimation, the effect of the change of solar activity is less than $10 \%$ of the daily fluctuations of the meteorological phenomena. Therefore, it is neglected for daily change of the meteorological phenomena. However, it can not be neglected for long period fluctuations.

The purpose of the present investigation is to confirm the stable periodicities which are expected from the author's hypothesis and to detect the effect of the change of the solar activity to the earth's atmosphere. It may be worth to refer one of the reason why such periodicities are examined. Because the effect of the change of the solar activity is very 
small, it is very difficult to confirm it by usual statistical analysis, such as lag correlation method. In regard to this point, method of harmonic analysis combined with the periodogram analysis is better, because by applying this method, full data contribute to the detection and detecting power is large.

Accordingly, the present investigation will proceed in the following schedule. 1st) Theoretical calculation of the tidal oscillation on the sun due to the planets and the determination of the periodicities. 2nd) Detection of the above periodicities in the change of the solar activity 3rd) Detection of the effect of the change of solar activity in the general circulation of the atmosphere. 4th) Investi- gation of the modulation of the periodicities due to the general circulation.

\section{Theory of the tidal oscillation on the sun}

As the start of our analysis, the tidal oscillation on the sun will be investigated. The results of the calculation of the tidal oscillation have been reported in the previous paper [6]. However, as there were some mistakes, revised results will be shown here.

As the orbital plane of the planets agrees roughly with the equatorial plane of the sun, the height $H$ of the tide on the sun is given by the following equation after famous theory of Darwin [7],

$$
\begin{aligned}
H=\sum & \frac{3}{2} \frac{M_{i}}{E}\left(\frac{a}{C_{i}}\right)^{3} a \cos ^{2} \varphi\left[\frac{1}{2} \cos \left(2 \omega_{s} t-2 \omega_{i} t+\Phi_{1}\right)\right. \\
& \left.+\frac{7}{4} e_{i} \cos \left(2 \omega_{s} t-2 \omega_{i} t+\Phi_{2}\right)+\frac{1}{4} e_{i} \cos \left(2 \omega_{s} t-\omega_{i} t+\Phi_{3}\right)\right] \\
+ & \sum \frac{3}{2} \frac{M_{i}}{E}\left(\frac{a}{C_{i}}\right)^{3} a I_{i} \sin 2 \varphi\left[\frac{1}{2} \cos \left(\omega_{s} t-2 \omega_{i} t+\Phi_{4}\right)\right. \\
& \left.+\frac{7}{4} e_{i} \cos \left(\omega_{s} t-3 \omega_{i} t+\Phi_{5}\right)+\frac{1}{2} \cos \left(\omega_{s} t+\Phi_{6}\right)\right] \\
+ & \sum \frac{3}{2} \frac{M_{i}}{E}\left(\frac{a}{C_{i}}\right)^{3} a\left(\frac{1}{2}-\frac{3}{2} \sin ^{2} \varphi\right)\left\{\frac{1}{3}+e_{i} \cos \left(\omega_{i} t+\Phi_{7}\right)\right\},
\end{aligned}
$$

where $M_{i}$ is the mass of the planet, $E$ the mass of the sun, $a$ the radius of the sun, $C_{i}$ the mean radius of the planet's orbit, $\omega_{i}$ the angular velocity of the planet's revolution referred to the fixed star system, $\omega_{s}$ angular velocity of the solar rotation referred to the fixed star system, $e_{i}$ the eccentricity of the orbit of the planet, $\Phi_{1}, \Phi_{2}, \cdots, \Phi_{7}$ constants, $I_{i}$ angle between the orbital plane of the palnet and the equatorial plane of the sun, suffix $i$ the indication of each planet.

The 1st group in the right-hand side of the above equation, expresses semidiurnal tide, the 2nd group diurnal tide and the 3rd group long periodic tide referred to the sun. Assuming that the period of the rotation of the sun referred to fixed stars is 25 days, the periods of the harmonics of the tidal oscillation are calculated as shown in table 1.

The amplitudes in the table 1 are indices expressed in the unit of

$$
\frac{3}{4} \frac{M_{E}}{E}\left(\frac{a}{C_{E}}\right)^{3} a
$$

Table 1. Harmonics of the tides on the sun.

\begin{tabular}{l|cc|cc}
\hline & \multicolumn{2}{|c|}{ Semidiural } & \multicolumn{2}{c}{ Diurnal } \\
& Amplitude & Period & Amplitude & Period \\
\hline Jupiter & 2.27 & 12.5 days & 0.23 & 25.2 days \\
Venus & 2.15 & 14.1 & 0.15 & 32.3 \\
Earth & 1.00 & 13.4 & - & - \\
Mercury & 0.97 & 17.5 & - & - \\
Mercury (Eccentricity) & 0.70 & 21.8 & - & - \\
Mercury (Long) & 0.20 & 88.0 & - & - \\
\hline
\end{tabular}


taken equal to 1.00 for the earth, which is about $0.16 \mathrm{~mm}$.

As the period of the solar rotation is small compared with the period of the planetary revolution round the sun, modulation of the semidiurnal tides occurs by the coupling of the semidiurnal tides due to various planets. In other wards beat oscillation occurs. It is expressed approximately by the oscillation of 13.5 day period with varing amplitude. Assuming $\Phi_{1}, \Phi_{2}, \cdots$, and $\Phi_{7}$ are 0 , the energy of such oscillation, or, in other wards, square of the amplitude of the oscillation is given by the following equation after some calculations:

$$
\begin{aligned}
A^{2}= & A_{J}^{2}+A_{V}^{2}+A_{E}^{2}+A_{M}^{2}+B_{M}^{2} \\
& +2 A_{J} A_{V} \cos 2\left(\omega_{V}-\omega_{J}\right) t \\
& +2 A_{J} A_{E} \cos 2\left(\omega_{E}-\omega_{J}\right) t \\
& +2 A_{J} A_{M} \cos 2\left(\omega_{M}-\omega_{J}\right) t \\
& +2 A_{V} A_{E} \cos 2\left(\omega_{E}-\omega_{V}\right) t \\
& +2 A_{V} A_{M} \cos 2\left(\omega_{M}-\omega_{V}\right) t \\
& +2 A_{E} A_{M} \cos 2\left(\omega_{M}-\omega_{E}\right) t \\
& +2 A_{J} B_{M} \cos 2\left(\omega_{M}-\omega_{J}\right) t \\
& +2 A_{V} B_{M} \cos \left(3 \omega_{M}-2 \omega_{V}\right) t \\
& +2 A_{E} B_{M} \cos \left(3 \omega_{M}-2 \omega_{E}\right) t \\
& +2 A_{M} B_{M} \cos \omega_{M} t
\end{aligned}
$$

where $A_{J}, A_{V}, A_{E}$ and $A_{M}$ are the amplitudes

Table 2. Periodicities of the energy of the semidiurnal tide.

\begin{tabular}{l|c|cc|c}
\hline \multirow{2}{*}{ Origin } & Amplitude & \multicolumn{2}{|c|}{ Period } & $\begin{array}{c}\text { Modulated } \\
\text { period }\end{array}$ \\
\hline$A_{J} A_{V} A_{E} A_{M} B_{M}$ & 12.2 & $\infty$ months $\infty 5$ days & $\infty$ years \\
$2 A_{J} A_{V}$ & 9.6 & 3.893 & 23.683 & 12.130 \\
$2 A_{J} A_{E}$ & 4.5 & 6.552 & 39.860 & 5.931 \\
$2 A_{J} A_{M}$ & 4.4 & 1.475 & 8.972 & 7.378 \\
$2 A_{V} A_{E}$ & 4.3 & 9.592 & 58.351 & 3.983 \\
$2 A_{V} A_{M}$ & 4.1 & 2.375 & 14.446 & 18.820 \\
$2 A_{E} A_{M}$ & 3.8 & 1.903 & 11.580 & 3.296 \\
$2 A_{J} B_{M}$ & 3.3 & 0.977 & 5.941 & 3.476 \\
$2 A_{V} B_{M}$ & 3.1 & 1.304 & 7.931 & 4.873 \\
$2 A_{E} B_{M}$ & 1.4 & 1.148 & 6.982 & 2.192 \\
$2 A_{M} B_{M}$ & 1.4 & 2.890 & 17.582 & 6.575 \\
\hline
\end{tabular}

of the semidiurnal tides due to the Jupiter, the Venus, the Earth and the Mercury respectively, $B_{M}$, the eccentricity tide due to the eliptic orbit of the Mercury. The other harmonics are neglected as they are very small.

Thus, the energy of semidurnal tides is expressed by the superposition of several harmonics. The amplitudes and the periods of the harmonics calculated from the above equation are shown in table 2.

We can apply similar treatment to the diurnal tides on the sun. The amplitudes of the diurnal tides are small, except that the diurnal tides due to the Jupiter and the Venus are significant. The amplitudes and

\begin{tabular}{|c|c|c|c|c|}
\hline \multirow[b]{2}{*}{ Origin } & \multicolumn{2}{|c|}{ Diurnal tides } & \multicolumn{2}{|c|}{$\begin{array}{c}\text { Energy of the } \\
\text { diurnal tide }\end{array}$} \\
\hline & $\begin{array}{l}\text { Ampli- } \\
\text { tude }\end{array}$ & Period & $\begin{array}{l}\text { Ampli- } \\
\text { tude }\end{array}$ & Period \\
\hline $\begin{array}{l}\text { Jup. } \\
\text { Mer. }\end{array}$ & 0.28 & 25.0 days & 0.39 & $\infty$ years \\
\hline Jupiter & 0.23 & 25.2 & 0.35 & 5.9311 \\
\hline Mercury & 0.15 & 32.3 & 0.25 & 0.30761 \\
\hline
\end{tabular}
the periods of the tidal oscillation due to the coupling of these tides are expressed by

$$
\begin{aligned}
H^{\prime}= & A_{J^{\prime}} \cos \left(\omega_{s} t-2 \omega_{s} t\right)+A_{J^{\prime}} \cos \omega_{s} t \\
& +A_{V^{\prime}} \cos \left(\omega_{s} t-2 \omega_{V} t\right)+A_{V^{\prime}} \cos \omega_{s} t
\end{aligned}
$$

$$
\begin{aligned}
= & \cos \left(\omega_{s} t+\phi_{1}\right)\left[2 A_{J^{\prime}}{ }^{2}+2 A_{V^{\prime}}{ }^{2}\right. \\
& +\left(2 A_{J^{\prime}}{ }^{2}+2 A_{J^{\prime}} A_{V^{\prime}}\right) \cos 2 \omega_{J} t \\
& +\left(2 A_{V^{\prime}}{ }^{2}+2 A_{J^{\prime}} A_{V^{\prime}}\right) \cos 2 \omega_{V} t \\
& \left.+\cos 2\left(\omega_{V}-\omega_{J}\right) t\right]^{1 / 2}
\end{aligned}
$$

Numerical values of the energy and the periods of such oscillation are calculated as shown in table 3 .

Table 3. Amplitudes and periods of the diurnal tides on the sun. 


\section{Harmonic analysis of the change of the solar activity}

\section{3.a) 25 day and 27 day periodicities in the change of the solar activity}

The 27 day periodicty in the magnetic disturbances caused by the transit of the sunspots across the central meridian of the solar disc is well known [8]. According to the theory of solar corpuscular radiation, such magnetic disturbances are caused by the corpuscular flow which is emmitted from the area of the sunspots. Hence, they appear at every 27 days time interval as the result of the solar rotation.

For the purpose of the confirmation of the above theory, 5-day mean $\mathrm{K}$-indices of the magnetic disturbances which are observed at Kakioka Observatory in Japan from 1950 to 1956 are analysed by harmonic analysis assuming the period of 27.5 days. As the result of the analysis, the amplitude for the $\mathrm{K}$-indices is found to be 4.5. On the other hand, if the time series of the $\mathrm{K}$-indices are at random, the expectancy of the amplitudes are calculated to be 2.2 because the standard deviation of the $\mathrm{K}$-indices is 24 and the number of data is 462 . Thus, the actual amplitude is larger than twice the expectancy. According to the theory of statistics [9] the probability of the occurrence of such a case is quite small being less than $2 \%$. Therefore, the existence of 27.5 days periodicity in $\mathrm{K}$-index is concluded with the risk less than $2 \%$.

This conclusion is confirmed also by the comparision of the phase angles of 27.5 days harmonics at different time intervals.

It must be remarked, however, that such periodicity can not be detected in the time

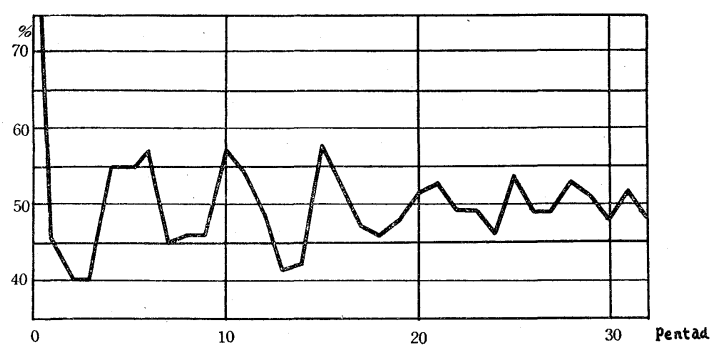

Fig. 1. Auto-correlation of 5 day mean sunspot numbers (1937-39). series of the sunspot number. As T. Asakura has pointed out, [10] 25 day periodicity predominates in the time series on the sunspot numbers. This is confirmed by taking the auto-correlations of 5 day mean sunspot numbers as shown in Fig. 1. (In the figure, the correlation is expressed by the percentage of the coincidence of the tendency in the change of sunspot number).

Thus, there is small but distinct difference between the periodicities in the change of $\mathrm{K}$-index and the sunspot number though they are similar indices of the solar activity. This is a complicated conclusion. However, if we consider that the number of sunspots is affected by the tidal oscillation on the sun and is independent of the solar orientation relative to earth, the periodicity of the sunspot number is 25 days. On the other hand, $\mathrm{K}$ index depends on the solar orientation, hence the periodicity of the $\mathrm{K}$-index coincides with the periods of the solar rotation against the earth, that is 27 days. Therefore, the contents in both indices are different though they are both the indices of the solar activity.

It is interesting that the 25-day periodicity predominates in the changes of the meteorological phenomena as will be seen later.

\section{3.b) Periodicity in the change of the sunspot number}

In the following harmonic analysis of the long-periodic variation of the solar activity, sunspot number is used as the index of the solar activity, because it is the only data available for a very long time interval indispensable for our analysis.

First, the mean amplitudes of the harmonics obtained by 24 term harmonic analysis of 2 month mean sunspot number, are shown in table 4 . The time interval of the analysis is from 1901 to 1948.

The mean amplitude of the harmonics decreases with the decrease of the period, however, weak maximum are found at 9.6, 6.85 and 4.8 months. As such maxima are weak, we cannot conclude at once that they are significant. However, such periodicities are expected from the standpoint of the tidal oscillation on the sun. 
Table 4. Mean amplitudes of the harmonics in the change of the sunspot numbers (1901-1948).

\begin{tabular}{|c|c|c|c|c|c|c|c|c|c|c|c|}
\hline \multirow[b]{2}{*}{ Period } & \multicolumn{11}{|c|}{ Months } \\
\hline & 48 & 24 & 16 & 12 & 9.6 & 8 & 6.85 & 6 & 5.32 & 4.8 & 4.37 \\
\hline Mean Amp. & 26.0 & 10.8 & 7.4 & 6.9 & 7.0 & 3.5 & 5.5 & 4.7 & 4.0 & 4.8 & 4.2 \\
\hline
\end{tabular}

\section{3.c) 2.890 month periodicity}

In the above analysis, the resolving power of the periodicity is weak. Therefore expected periodicity is analysed by harmonic analysis for long time interval. The method of the analysis of 2.89 month periodicity will be explained as an example. Here 2.89 month period is that of the tides on the sun due to the revolution of the Mercury round the sun.

First, the time series of the sunspot number is divided into sequences of about 4 year period, that is $2.89 \times 18=52.02$ months. The amplitude and the phase of 2.89 month harmonics are calculated by usual harmonic analysis for each time interval as above. The results of the calculation are showu in table 5 . If the 2.89 month harmonic is real, the phase angle must remain constant for the change of the analysed time intervals, because they are decided to be integral multiples of the period to be detected.

The results of the calculation are not so simple and our expectation seems to have failed at a first glance. However, after some researches, we found that the phase angle of 2.89 month periodicity was conserved if we take into account the change of the well known 11 year cycle of sunspots.

Analysed phase angles are classified into two groups, that is, those in the increasing or stationary stage and those in the decreasing stage of the 11 years cycle of the sunspot number, and they are expressed by polar coordinate in Fig. 2 a. The length of the vector is the amplitude and the angle between the vector and the abscissa is the phase angle of the harmonics. Small white circles and black circles indicate stationary or increasing and decreasing stage of the 11 year cycle respectively. We found that the almost all vectors in these two stage are in opposite direction, one about $220^{\circ}$, the other about $20^{\circ}$. Such a character is improbable if the change of the sunspot number is at random.

There may arise a question that, the systematic phase difference revealed in the phase diagram is due to the systematic error of the analysis for increasing or decreasing stage of the 11 year cycle, which is the most predominant change in the sunspot number. However, rough estimation shows that periods shorter than 3 months are not affected by the systematic error, and even for the period of 10 months to be referred to later, such error does not amount to a magnitude so as to change the above conclusion.

Table 5. 2.89 month periodicity in sunspot number. (+ increasing, - decreasing, S stationary stage of 11 years cycle)

\begin{tabular}{c|cc|cc}
\hline \multirow{2}{*}{ Time } & $\begin{array}{c}\text { Ampli- } \\
\text { tude }\end{array}$ & Phase & Tendency & $\begin{array}{c}\text { Mean of sun- } \\
\text { spot }\end{array}$ \\
\hline \multirow{1}{*}{1900} & 1.4 & $220^{\circ}$ & + & 20 \\
& 3.3 & 250 & $\mathrm{~S}$ & 30 \\
& 1.9 & 350 & - & 20 \\
1913 & 0.9 & 220 & + & 35 \\
& 2.2 & 20 & - & 60 \\
& 0.03 & 270 & + & 18 \\
1926 & 1.1 & 100 & $\mathrm{~S}$ & 75 \\
& 0.6 & 90 & - & 15 \\
& 4.1 & 340 & + & 80 \\
& 2.2 & 340 & - & 55 \\
& 3.9 & 220 & + & 65 \\
& 1.0 & 60 & - & 120 \\
\hline
\end{tabular}

For the statistical test of the significance of the above character, $\chi^{2}$-test may be convenient. In 5 phase angles of the harmonics at the increasing stage of the 11 year cycle of the sunspot number as shown in table 5 , 4 phase angles of the harmonics fall in $3 \mathrm{rd}$ quadrant, that is, between $180^{\circ}$ and $270^{\circ}$, and one in 4 th quadrant, that is, between $270^{\circ}$ and $360^{\circ}$. If there is no real periodicity, the probability that the phase angle falls at certain quadrant is equal. In our case the expectancy of the cases in each quadrant is $5 / 4=1.25$, and the expectancy of $\chi^{2}$ is 4 , while the calculated $\chi^{2}$ is 8.6.

According to the table made by Fisher (11), 
the conclusion that the phase angle is not at random is significant with the risk of $5 \%$ as the degree of freedom is $4-1=3$. We get similar conclusion for the other phase angles.

We may conclude therefore that the 2.89 month periodicity in the change of the sunspot number is real though the phase angles are converted at increasing and decreasing stage of the 11 year cycle of the sunspot number.

\section{3.d) 3.896 month periodicity}

The most important tide-generating force on the sun is produced by the Jupiter and the Venus. Hence the 3.896 month periodicity, that is half of the synodic period of the Jupiter and the Venus, is expected to predominate in the change of the solar activity.

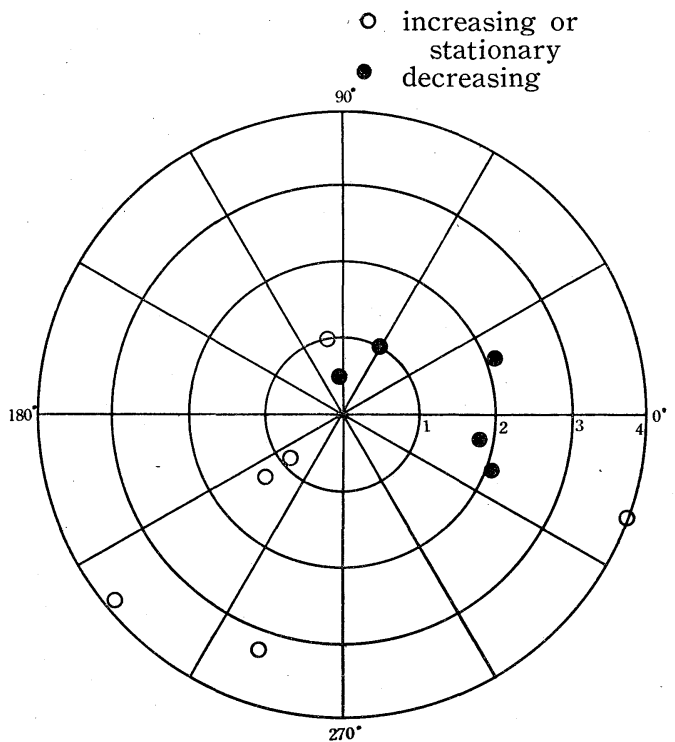

Fig. 2a. 2.89 month periodicity (1900-1954).

Accordingly, this periodicity is examined by the similar method as above for the observational data of sunspot number from 1860 to 1950.

Harmonic vectors analysed for 4 years time interval are shown in Fig. $2 \mathrm{~b}$, where white small circles represent the stage of much sunspots in the 11 year cycle (mean sunspot number is larger than 50) and black small circles the stage of few sunspots (less than 49).

We see that these two kinds of the points are distributed almost on opposite sides,

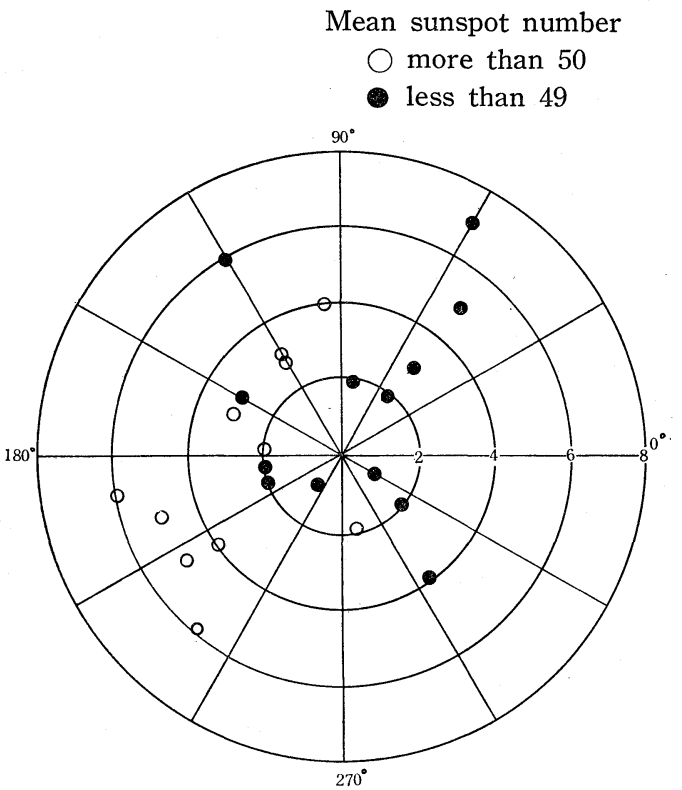

Fig. 2 b. 3.896 month periodicity (1860-1954).

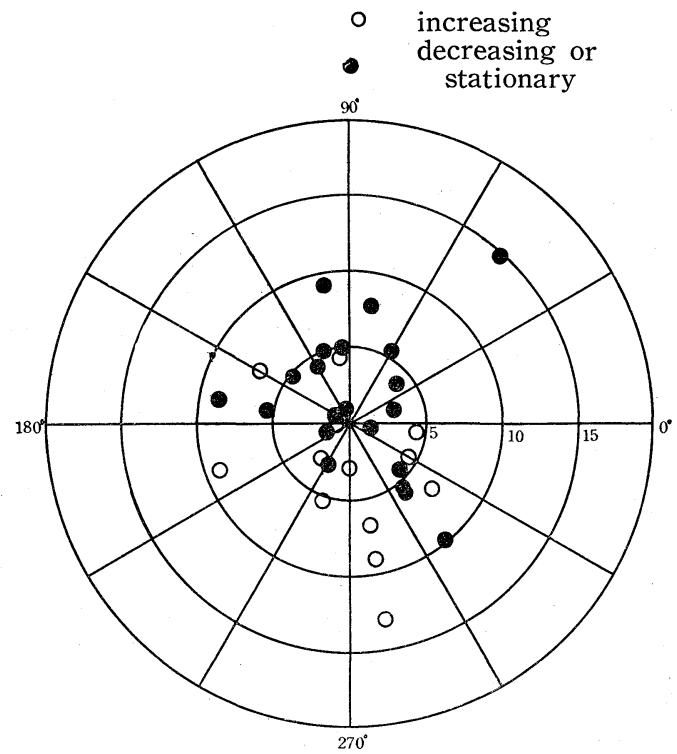

Fig. 2 c. 9.592 month periodicity (1814-1954)

hence such periodicity may be considered to be real. The significance of the above conclusion is proved to be true by means of the statistical tests.

\section{3.e) 9.592 month periodicity}

Next, the 9.592 month periodicty, that is, half of the synodic period of the Venus and the Earth, will be examined on the data from 1814 to 1954 . The harmonic vectors analysed 
for 4 year time intervals are shown in Fig. $2 \mathrm{c}$ by classifying the two stages of the 11 year cycle of sunspots, where white circles represent the increasing stage and black circles the decreasing or stationary stage of the 11 year cycle of sunspots.

We may conclude that the periodicity is real also in this case.

Thus, the periodicities which are expected from the standpoint of the tidal oscillation on the sun is detectable. Hewever, the phase angles of the harmonics depend on the stage of the 11 year cycle of sunspots. Therefore, the tidal periods cannot be detected if the change of the phase is not taken into account in the harmonic analysis. This may be one of the reason that such periodicities have not been accepted fully by astronomers and meteorologists.

4. Harmonic analysis of some of the meteorological elements related with the periodicities in the change of the solar activity

\section{4.a) Scheme of the analysis}

The purpose of this section is to find out the relation between the periodicities in the change of the solar activity and those in the meteorological phenomena on the earth.

If the meteorological phenomena on the earth are affected by the change of solar activity, the same periodicities must be detected both in the change of solar activity and in the change of meteorological elements on the earth. On the other hand, if we can detect the same periodicities in the meteorological elements with that in solar activity, we may conclude that the solar activity affects the meteorological phenomena.

Accordingly the amplitudes and the phase angles of a certain harmonic are calculated for the time series of some meteorological elements and for that of solar activity in the same time interval, assuming the periodicities which are expected from the tidal oscillation on the sun. Next, the calculated amplitudes and the phase angles in the meteorological elements and in the solar activity are compared with each other. If the meteorological elements are affected by the change of solar activity, both amplitudes and phase angles will change in parallel and the difference of the phase angles will be kept constant. This is the fundamental procedure of the analysis.

\section{4.b) 25 day periodicity}

The present author and T. Asakura have analysed 25 day periodicity in the meteorological elements and the solar activity and they showed that such periodicity may originate from the change of the solar radiation with the rotation of the sun. The elements treated in that report are, however, restricted only to surface data of the meteorological elements and the sunspot number. Therefore, the relation will be reexamined by adding $500 \mathrm{mb}$ height, $\mathrm{K}$-index (index of corpuscular flow) and others.

The amplitudes and the phase angles of 25 day harmonics are calculated on the data of successive 25 pentads (almost 125 days) of the sunspot number, $\mathrm{K}$-index, $500 \mathrm{mb}$ height anomalies at $40^{\circ} \mathrm{N} 140^{\circ} \mathrm{E}$, and $70^{\circ} \mathrm{N} 120^{\circ} \mathrm{E}$, ratio of the areal precipitation for River Kiso against the normal for the time interval 19491953.

The results of the calculation are shown in table 6 .

Now the frequency of the difference in phase angles between the harmonics of sunspot number and $500 \mathrm{mb}$ height anomaly at $40^{\circ} \mathrm{N} 140^{\circ} \mathrm{E}$ is as follows:

If the phase angles of 25 day harmonics for the sunspot number and the $500 \mathrm{mb}$ height anamoaly are independent of each other, the frequency of the difference between both phase angles is expected to be uniform and $\chi^{2}$ is expected to be 4 . On the other hand, calculated $\chi^{2}$ is 9 , therefore the distribution is never uniform. After the statistical significance test, the present assumption is rejected with the risk of the probability of less than 5\%. Therefore, 25 day periodicity in the sunspot number and $500 \mathrm{mb}$ height anomaly are correlated with each other.

Next the amplitudes for the sunspot number and that for the areal precipitation varies in parallel.

Thus we may conclude that the 25 day periodicity in the change of sunspot number and of the meteorological elements are correlated with each other. This agrees well 
Table 6. 25 day harmonics.

(A Amplitude, $\varphi$ phase angle of the harmonic)

\begin{tabular}{|c|c|c|c|c|c|c|c|c|c|c|}
\hline \multirow[b]{2}{*}{ Year } & \multicolumn{2}{|c|}{ Sunspots } & \multicolumn{2}{|c|}{$\mathrm{K}$-index } & \multicolumn{4}{|c|}{$500 \mathrm{mb}$ anomaly } & \multicolumn{2}{|c|}{ Areal percipitation } \\
\hline & A & $\varphi$ & A & $\varphi$ & $\begin{array}{c}40^{\circ} \mathrm{N} \\
\mathrm{A}\end{array}$ & $\begin{array}{c}140^{\circ} \mathrm{E} \\
\varphi\end{array}$ & $\begin{array}{c}70^{\circ} \mathrm{N} \\
\mathrm{A}\end{array}$ & $\begin{array}{c}120^{\circ} \mathrm{E} \\
\varphi\end{array}$ & A & $\varphi$ \\
\hline \multirow[t]{3}{*}{1949} & 49 & $20^{\circ}$ & & & $56 \mathrm{ft}$ & $30^{\circ}$ & $95 \mathrm{ft}$ & $170^{\circ}$ & 0.37 & $50^{\circ}$ \\
\hline & 35 & 180 & & & 29 & 100 & 82 & 290 & 19 & 280 \\
\hline & 10 & 210 & & & 37 & 260 & 118 & 280 & 24 & 80 \\
\hline \multirow[t]{3}{*}{1950} & 54 & 280 & 6 & $320^{\circ}$ & 85 & 170 & 109 & 270 & 51 & 130 \\
\hline & 8 & 320 & 7 & 310 & 52 & 320 & 122 & 30 & 24 & 100 \\
\hline & 26 & 140 & 15 & 40 & 104 & 60 & 94 & 270 & 40 & 310 \\
\hline \multirow[t]{3}{*}{1951} & 25 & 280 & 2 & 210 & 86 & 240 & 149 & 290 & 30 & 350 \\
\hline & 30 & 30 & 16 & 270 & 45 & 250 & 135 & 300 & 22 & 90 \\
\hline & 8 & 310 & 15 & 40 & 73 & 30 & 72 & 110 & 20 & 80 \\
\hline \multirow[t]{3}{*}{1952} & 6 & 340 & 15 & 290 & 108 & 50 & 196 & 160 & 45 & 60 \\
\hline & 25 & 320 & 11 & 10 & 104 & 350 & 101 & 130 & 47 & 350 \\
\hline & 28 & 40 & 7 & 20 & 40 & 50 & 156 & 180 & 48 & 50 \\
\hline \multirow[t]{2}{*}{1953} & 16 & 150 & 14 & 0 & 71 & 220 & 131 & 300 & 18 & 250 \\
\hline & 11 & 250 & 15 & 80 & 23 & 170 & 65 & 270 & 46 & 260 \\
\hline
\end{tabular}

Table 7.

\begin{tabular}{c|cccc}
\hline $\begin{array}{c}\text { Phase dif- } \\
\text { ference }\end{array}$ & $0-90^{\circ}$ & $90-180^{\circ}$ & $180-270^{\circ}$ & $270-360^{\circ}$ \\
\hline Frequency & 8 & 0 & 2 & 4 \\
\hline
\end{tabular}

with the result of our former investigation. We can not find, however, any correlations between $\mathrm{K}$-index and the meteorological elements.

\section{4.c) 72 day periodicity}

The 72 day periodicity in the pressure change has been investigated already by $\mathrm{L}$. Weickmann and others, [12], and it is also found in the change of solar activity. From the standpoint of the tidal oscillation on the sun, such a periodicity is 14.4465 pentads (one pentad is equal to 365.24 days $\div 73$ ) which is one half of the synodic period of the Venus and the Mercury.

The amplitude and the phase angles of this harmonics in the change of sunspot number, $\mathrm{K}$-index and $500 \mathrm{mb}$ height anomaly at $60^{\circ} \mathrm{N}$ $100^{\circ} \mathrm{N}$ are calculated for the data from 1947 to 1955 . Next the calculated phase angles of the harmonics are classified into two groups according to the stage of the 11 year cycle of the spots, and they are plotted in the phase diagram as shown in Fig. 3.

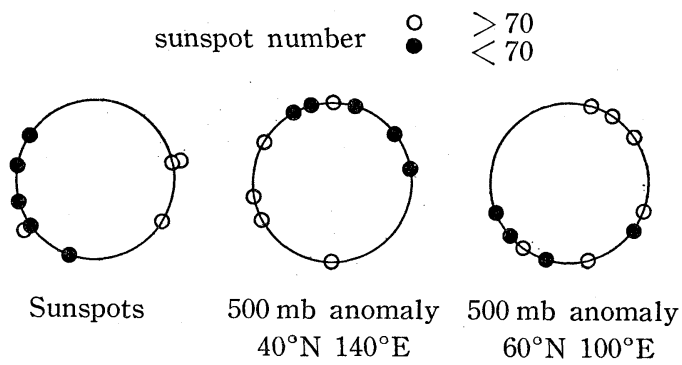

Fig. 3. 72 day periodicity.

The phase angles of the harmonics in sunspots as well as $500 \mathrm{mb}$ height fall one side of the phase diagram when the annual mean number of the sunspot is less than 70 . Therefore, 72 day periodicity is statistically significant. It may be concluded also that the phase angles of the harmonics of $500 \mathrm{mb}$ height anomalies at $60^{\circ} \mathrm{N} 100^{\circ} \mathrm{E}$ and at $40^{\circ} \mathrm{N}$ $140^{\circ} \mathrm{E}$ are in opposite phase and the former coincides roughly with the phase of the sunspot number.

It may be concluded further that the $\mathrm{K}$ index affects slightly to the meteorological elements.

\section{4.d) 2.89 month periodicity}

The 3 month periodicity in the atmospheric pressure has been found by S. T. Pagava [13] and sometimes we experience such periodici- 
ty predominating in some of the meteorological elements. This periodicity seems to correspond to the 2.89 month periodicity due to the tide on the sun produced by the Mercury. The amplitudes and the phase angles of the periodicity in the sunspot number and $500 \mathrm{mb}$ height anomaly at $60^{\circ} \mathrm{N} 100^{\circ} \mathrm{E}$ are calculated by the data observed form 1947 to 1956 .

Though the time interval of the analysis is not enough to fix the following conclusion, we may conclude that the amplitudes of the harmonics in the change of the sunspot number and the $500 \mathrm{mb}$ height anomaly for successive time intervals change in parallel and the phase difference between them remains almost the same.

\section{4.e) 9.592 month periodicity}

The 9.592 periodicity is a half of the syno- dic period of the Venus and the Earth. Though such periodicity has not been recognized in the meteorological phenomena in the past so far as I know, the present analysis proves that such periodicity predominates in the meteorological elements. As the period is long, it is difficult to confirm it by the harmonic analysis of the $500 \mathrm{mb}$ height anomaly owing to the shortness of the observation period. Therefore 9.592 month harmonic is analysed for the monthly mean pressure anomaly at Tokyo and the temperature anomaly at Sapporo. Namely the amplitudes and the phase angles of 9.592 month harmonics are calculated by succesive 4 year time intervals for the period from 1879 to 1953.

Frequency distribution of the phase differences between the sunspot number and the

Table 8. Frequency of phase differences.

\begin{tabular}{l|cccc}
\hline \multicolumn{1}{c|}{ Phase angle } & $-45^{\circ}-45^{\circ}$ & $45^{\circ}-135^{\circ}$ & $135^{\circ}-225^{\circ}$ & $225^{\circ}-315^{\circ}$ \\
\hline Pressure (Tokyo) & 2 & 3 & 7 & 1 \\
Temperature (Sapporo) & 3 & 1 & 1 & 6 \\
\hline
\end{tabular}

pressure as well as the temperature is shown in table 8 , excluding the cases of very small amplitudes. The constancy of the phase difference is good and it is statistically significant with the risk less than $10 \%$.

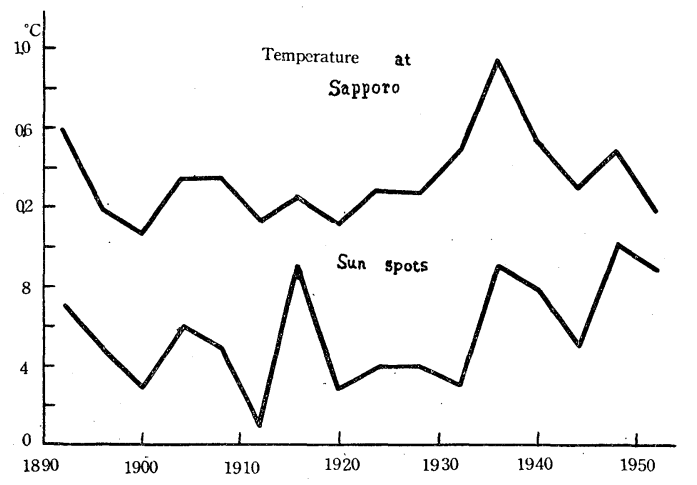

Fig. 4. Time change of the amplitudes of 9.6 month harmonics.

Next it is proved that the amplitudes of the temperature change are in high correlation with that of the sunspot number as shown in Fig. 4. The correlation coefficient is $0.61 \pm 0.20$.

It may be noticed that the 2nd harmonic of this periodicity, that is the 4.8 month periodicity predominates in the change of sunspot number as well as in the change of atmospheric pressure. For instance, 5 month periodicity is well known in U.S.S. R. [13]. The present author and T. Asakura [14] have analysed 5 month periodicity in the pressure pattern of the northern hemisphere and concluded that the periodicity seems to occur by the effects of the solar activity.

\section{Modification of the periodicity due to the annual change of the general circulation}

The annual change of the general circulation is large in the earth's atmosphere. Hence, there is a possibility that the modulation of the periodicity in the earth's atmosphere occur by the effect of the solar activity. Though the theoretical consideration of such modulation is quite difficult, it will be examined by a simple model. Let the temperature distribution on the earth be given by the following equation of heat budget: 


$$
\frac{\partial \theta}{\partial t}=\frac{K}{R^{2} \cos \varphi} \frac{\partial}{\partial \varphi}\left(\cos \varphi \frac{\partial \theta}{\partial \varphi}\right)+J(\varphi)-\sigma \theta^{4}
$$

where $\theta$ is the mean temperature of the atmosphere at the latitude circle $\varphi, K$ the large scale austausch coefficient, $R$ the radius of the earth, $J(\varphi)$ the effective income radiation at $\varphi, \sigma \theta^{4}(\varphi)$, the effective outgoing radiation at $\varphi$. The earth is in thermal equilibrium as a mean for long time interval, and the above equation is rewritten as follows.

$$
\begin{aligned}
& \frac{\partial \Delta \theta}{\partial t}-\frac{K_{0}}{R^{2} \cos \varphi} \frac{\partial}{\partial \varphi}\left(\cos \varphi \frac{\partial \Delta \theta}{\partial \varphi}\right)+4 \sigma \theta_{0} 4 \frac{\Delta \theta}{\theta_{0}} \\
& =\frac{\Delta K}{R^{2} \cos \varphi} \frac{\partial}{\partial \varphi}\left(\cos \varphi \frac{\partial \theta_{0}}{\partial \varphi}\right)+J_{0} \frac{\Delta J}{J_{0}} \\
& \quad+\Delta K \frac{\cos \omega_{0} t}{R^{2} \cos \varphi} \frac{\partial}{\partial \varphi}\left(\cos \varphi \frac{\partial b}{\partial \varphi}\right)
\end{aligned}
$$

where $K_{0}$ is the mean austausch coefficient, $\theta_{0}$ the mean temperature, $J_{0}$ the mean effective income radiation, $\Delta$ denotes anomaly from the mean, $b$ the amplitude of the annual change of the temperature, $\omega_{0}$ the angular velocity of 1 year cycle.

Assuming further that $\Delta K$ and $\Delta J$ change cyclicial with the periodicity in the solar activity, that is,

$$
\Delta K \propto \sin \omega t, \quad \Delta J \propto \sin \omega t,
$$

following terms are derived from the last term in the right hand side of the equation.

$$
\begin{aligned}
& \sin \omega t \cos \omega_{0} t \\
& \quad=\frac{1}{2}\left\{\sin \left(\omega+\omega_{0}\right) t+\sin \left(\omega-\omega_{0}\right) t\right\}
\end{aligned}
$$

Thus new periodicity arises from the coupling of the annual change of the general circulation and the periodicity of the solar radiation. Such modified periodicities are calculated as shown in table 9 .

Table 9. Modulation of the periodicity.

\begin{tabular}{l|ll}
\hline$\frac{2 \pi}{\omega}$ & $\frac{2 \pi}{\omega+\omega_{0}}$ & $\frac{2 \pi}{\omega-\omega_{0}}$ \\
\hline 9.592 month & 5.331 month & 47.800 month \\
6.552 & 4.238 & 14.434 \\
3.893 & 2.939 & 5.762 \\
$1.475^{3}$ & 1.314 & 1.682 \\
2.375 & 1.982 & 2.961 \\
2.890 & 2.329 & 3.807 \\
\hline
\end{tabular}

Of cource, the amplitudes of the modulated periodicities are expected to be small. However, if the present expectation is right, the periodicity in Table 9 must be detected in the time change of the meteorological elements. The present author has shown in the former paper that 4-year periodicity predominates in the various meteorological elements. This seems to correspond to 47.8 month periodicity in Table 9 .

The amplitudes and phase angles of the 5.33 month periodicity in the pressure change at Tokyo were also calculated for successive 4 year time intervals from 1883 to 1954 . The phase angles of the harmonics at the increasing stage and the decreasing or stationary stage of the 11 year cycle of the sunspot remain almost constant respectively as shown in Fig. 5. This is proved to be significant with the risk less than $5 \%$.

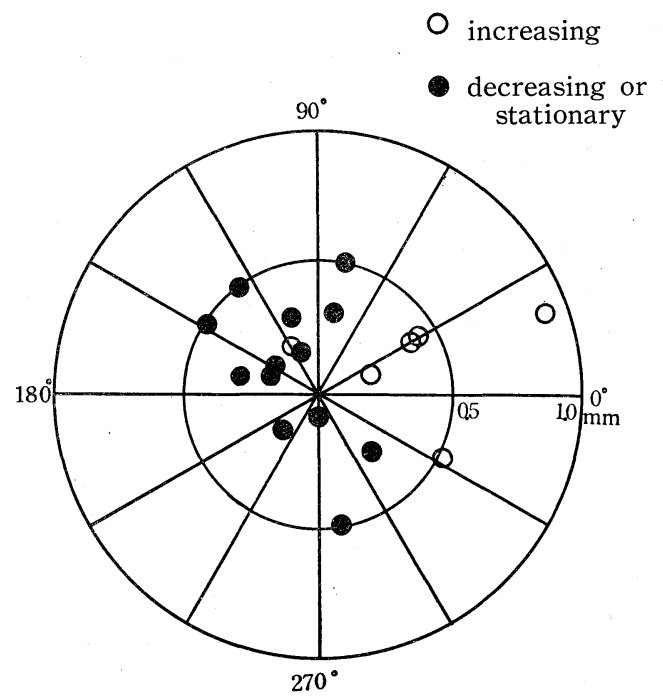

Fig. 5. 5.33 month periodicity of the pressure at Tokyo (1883-1954).

Thus, it is quite probable that such periodicities appear in the meteorological elements though the other periodicities in Table 9 is not yet detected.

\section{Conclusion}

From these investigations, it may be concluded that the stable harmonics exist in the change of the solar activity though their amplitudes are very small. This is expected from the tidal oscillation on the sun. Phase 
angles of the harmonics, however, depend on the stage of the 11 year cycle of the sunspot number. It may be concluded further that there are corresponding harmonics in the change of some of the meteorological elements. The amplitudes of such harmonics are very small, the amplitude of the harmonics in the change of $500 \mathrm{mb}$ height anomaly is estimated to be of the order of $50 \mathrm{ft}$, that is $0.5 \%$ of the absolute height. Therefore, it is negligible for the daily change of meteorogical elements, but it can not be neglected for the change of long periodic change, for instance, one month change or yearly change. Because, random fluctuations of daily disturbance will die out by the smoothing, while the long periodic change of the harmonics of our concern is systematic and suffers little change by the smoothing.

The mode of the atmospheric oscillation due to the change of the solar activity is complicated and there are few researches on this problem. It seems to me that such oscillations occur with the exchange of heat energy between high and low latitudes. Recent investigations by Y. Arai [15], T. Katayama and T. Asakura [16], suggest that the increase of the solar activity is accompanied by the increase of blocking action, in other wards, the increase of the exchange of heat energy.

Significant correlations are found between the sunspot number and the meteorological elements but only few correlations between $\mathrm{K}$-index and the meteorological elements. These results seem to contradict with the importance of the corpuscular flow as the solar effect to the atmosphere. But the physical process of the effect of the change of the solar activity to the earth's atmosphere is not yet clear, and the above conclusion can not be affirmed without further researches.

Analytical and physical investigations on these problems are wanted in future.

\section{References}

1. Clayton H. H. (1934): World Weather and Solar Activity, Smith. Mis. Coll. 89, 359-376.

2. Clayton H. H. (1935): Long Period Weather Change and Method of Forecasting. Month. Weath. Rev. 64, 359-376.

3. Craig R. A. (1952): Surface-pressure Variations following Geomagnetically Disturbed days and Geomagnetically Quiet days, Jour. Met. 9, $126-138$.

4. Shapiro R. (1956): Further Evidence of a Solar Weather Effect, Jour. Met. 13, 335-340.

5. Roberts W. O. (1956): Cross-field Seminar on Possible Responses of Terrestrial Atmospheric Circulation to Changes in Solar Activity. Bull. Amer. Met. Soc. 37, 477-479.

6. Takahashi K. (1957): Studies on the Seasonal Weather Forecasting (V)-Tidal Oscillation on the Sun as the Cause of the Periodicities in the Meteorological Phenomena Pap. Met. Geophy. 7, 347-357.

7. Darwin G. H. (1907): Oceanic Tides (Scientific Papers Vol. I).

8. Chapman S. and Bartels J. (1940): Geomagnetism Vol. I (Oxford, Clarenden Press).

9. Conrad V. and Pollak L. W. (1950): Methods in Climatology (Harvard Univ. Press.)

10. Asakura T. (1954): 25-day period in Meteorological Phenomena and in Solar Activity. Jour. Met. Res. 6, 165-171.

11. Fisher R. A. (1950): Statistical Methods for Research Workers (Oliver and Body)

12. Oehmisch W. (1940): Analyse einer 72-tägigen Luftdruck-welle in den Wintern 1923/24 und 1924/25. Veröff. Geophys. Inst. d. Univ. Leipzig. 12, 175-225.

13. Pagava S. T. and others (1940): Basic Principles of the Synoptic Method of Longrange Weather Forecasting, Leningrad. U.S.S.R. Hydrometoro. Publ. House.

14. Takahashi K. and Asakura T. (1954): Relation of the Sun and the Weather. Jour. Met. Soc. Jap. 32, 290-302.

15. Arai Y. (1958): Characteristics of Long Waves in Westerlies related to Solar Activity Jour. Met. Soc. Jap. 36, 46-54.

16. Katayama H. and Asakura T. (1958): A Possible Effect of Solar Activity to the General Circulation of the Atmosphere.

(to be published in Pap. Met. Geophys.) 